\title{
Impulsive fractional quantum Hahn difference boundary value problems
}

Jessada Tariboon ${ }^{1 *}$, Sotiris K. Ntouyas ${ }^{2,3}$ and Bundit Sutthasin ${ }^{1}$

"Correspondence:

jessada.t@sci.kmutnb.ac.th

${ }^{1}$ Intelligent and Nonlinear Dynamic

Innovations Research Center,

Department of Mathematics,

Faculty of Applied Science, King

Mongkut's University of Technology

North Bangkok, Bangkok, Thailand

Full list of author information is

available at the end of the article

\begin{abstract}
In this paper, we study the existence and uniqueness of solutions for two classes of boundary value problems for impulsive Caputo type fractional Hahn difference equations, by using the Banach contraction mapping principle and the nonlinear alternative of Leray-Schauder. The obtained results are well illustrated by examples.
\end{abstract}

MSC: Primary 39A10; 39A13; secondary 39A70

Keywords: Hahn difference equations; Boundary value problems; Existence; Uniqueness; Fixed point theorems

\section{Introduction and preliminaries}

Our purpose of this paper is to establish the existence and uniqueness results for two impulsive fractional Hanh difference boundary value problems. More precisely, we consider the first boundary value problem of order $v_{k}, 0<v_{k} \leq 1$,

$$
\left\{\begin{array}{l}
{ }_{t_{k}}^{c} D_{q_{k}, \omega_{k}}^{v_{k}} x(t)=f(t, x(t)), \quad t \in J_{k}, k=0,1,2, \ldots, m, \\
\Delta x\left(t_{k}\right)=\varphi_{k}\left(x\left(t_{k}^{-}\right)\right), \quad k=1,2, \ldots, m, \\
\xi_{1} x(0)+\xi_{2} x(T)=\xi_{3},
\end{array}\right.
$$

and the second boundary value problem of order $v_{k}, 1<v_{k} \leq 2$,

$$
\left\{\begin{array}{l}
t_{k} D_{q_{k}, \omega_{k}}^{v_{k}} x(t)=f(t, x(t)), \quad t \in J_{k}, k=0,1,2, \ldots, m, \\
\Delta x\left(t_{k}\right)=\varphi_{k}\left(x\left(t_{k}^{-}\right)\right), \quad k=1,2, \ldots, m, \\
t_{k} D_{q_{k}, \omega_{k}} x\left(t_{k}^{+}\right)-t_{k-1} D_{q_{k-1}, \omega_{k-1}} x\left(t_{k}^{-}\right)=\varphi_{k}^{*}\left(x\left(t_{k}^{-}\right)\right), \quad k=1,2, \ldots, m, \\
x(0)=\eta_{1}, \quad t_{m} D_{q_{m}, \omega_{m}} x(T)=\eta_{2},
\end{array}\right.
$$

where ${ }_{t_{k}}^{c} D_{q_{k}, \omega_{k}}^{v_{k}}$ is the fractional quantum Hahn difference operator of Caputo type, $0<$ $q_{k}<1, \omega_{k} \geq 0, k=0,1,2, \ldots, m, f: J \times \mathbb{R} \rightarrow \mathbb{R}, J=[0, T], \varphi, \varphi_{k}^{*}: \mathbb{R} \rightarrow \mathbb{R}, k=1,2, \ldots, m$, are given functions, $\Delta x\left(t_{k}\right)=x\left(t_{k}\right)-x\left(t_{k}^{-}\right),{ }_{k} D_{q_{k}, \omega_{k}}$ is the first order quantum Hahn difference operator on interval $J_{k}, k=0,1,2, \ldots, m$, and given constants $\xi_{1}, \xi_{2}, \xi_{3}, \eta_{1}, \eta_{2} \in \mathbb{R}$.

The $q$-calculus appeared as a connection between mathematics and physics, especially, in elementary particle physics, which have used quantum numbers to present the discrete

(c) The Author(s) 2019. This article is distributed under the terms of the Creative Commons Attribution 4.0 International License (http://creativecommons.org/licenses/by/4.0/), which permits unrestricted use, distribution, and reproduction in any medium, provided you give appropriate credit to the original author(s) and the source, provide a link to the Creative Commons license, and indicate if changes were made. 
values of energy levels in atoms. In 1910, Jackson [1] introduced the notion of $q$-derivative as

$$
D_{q} f(t)= \begin{cases}\frac{f(t)-f(q t)}{t(1-q)}, & t \neq 0, \\ f^{\prime}(0), & t=0,\end{cases}
$$

provided that $f^{\prime}(0)$ exists. He also was the first to develop $q$-calculus and $q$-difference equations in a systematic way. The book by Kac and Cheung [2] covers many of the fundamental aspects of quantum calculus and also $q$-special functions. The $q$-calculus has many applications in mathematical areas such as orthogonal polynomials, basic hypergeometric functions, combinatorics, the calculus of variations, quantum mechanics, and the theory of relativity. Some recent results in quantum calculus can be found in [3-9] and the references cited therein.

Hahn [10] introduced his difference operator $D_{q, \omega}$ as

$$
D_{q, \omega} f(t)= \begin{cases}\frac{f(q t+\omega)-f(t)}{t(q-1)+\omega}, & t \neq \omega_{0}, \\ f^{\prime}\left(\omega_{0}\right), & t=\omega_{0}\end{cases}
$$

provided that $f$ is differentiable at $\omega_{0}$, where $q \in(0,1)$ and $\omega \geq 0$ are fixed. Here $f$ is defined on an interval $I \subseteq \mathbb{R}$ containing $\omega_{0}:=\omega /(1-q)$. The Hahn difference operator unifies (in the limit) the two most well known and used quantum difference operators: the Jackson $q$-difference derivative $D_{q}$, where $q \in(0,1)$, defined by (3), for $\omega=0$, and the forward difference $D_{\omega}$ for $q \rightarrow 1$, defined by

$$
D_{\omega} f(t)=\frac{f(t+\omega)-f(t)}{\omega}
$$

where $\omega>0$ is a fixed constant. The Hahn difference operator is a successful tool for constructing families of orthogonal polynomials and investigating some approximation problems (cf. [11-14]). For some recent results on the boundary value problems of Hahn difference equations we refer to [15-19] and references therein.

Let us emphasize that the definition (3) does not remain valid for impulse points $t_{k}$, $k \in \mathbb{Z}$, such that $t_{k} \in(q t, t)$. For example, let $[0, T], T>4$ be a dense interval and $t=2$ be an impulsive point, i.e., $f\left(2^{+}\right) \neq f\left(2^{-}\right)$. Then we have $D_{1 / 2} f\left(4^{+}\right) \neq D_{1 / 2} f\left(4^{-}\right)$, which implies that $D_{1 / 2} f(4)$ does not exist. On the other hand, this situation does not arise for impulsive equations on $q$-time scales $\left\{0, \ldots, q^{2} t, q t, t\right\}$, as the domains consist of isolated points covering the case of consecutive points of $t$ and $q t$ with impulsive points $t_{k} \notin(q t, t)$. Due to this reason, the subject of impulsive quantum difference equations on dense domains could not be studied. In [20], the authors modified the classical quantum calculus on $[a, b]$ by defining

$$
{ }_{a} D_{q} f(t)= \begin{cases}\frac{f(t)-f(q t+(1-q) a)}{(1-q)(t-a)}, & t \neq a, \\ \lim _{t \rightarrow a a} D_{q} f(t), & t=a .\end{cases}
$$

Observe that if $t_{k}, k=1,2, \ldots$, are impulse points with $f\left(t_{k}^{+}\right)=f\left(t_{k}\right)$, then, by setting $[a, b)=\left[t_{k}, t_{k+1}\right)$, there is no impulse point in $[a, b)$. With the help of definition (6), a se- 
ries of impulsive quantum initial and boundary value problems were studied. We refer the interested reader to the recent monograph [21] for details.

In [22], the authors defined a quantum shifting operator by

$$
{ }_{a} \Phi_{q}(m)=q m+(1-q) a
$$

$m, a \in \mathbb{R}$ with $m \geq a$. Then the $q$-derivative of a function $f$ on an interval $[a, b]$ in (6) can be rewritten as

$$
{ }_{a} D_{q} f(t)= \begin{cases}\frac{f(t)-f\left(\Phi_{q}(t)\right)}{t-a \Phi_{q}(t)}, & t \neq a, \\ \lim _{t \rightarrow a a} D_{q} f(t), & t=a .\end{cases}
$$

Now, we consider the interval $[a, b] \subseteq \mathbb{R}$, the quantum numbers $0<q<1, \omega \geq 0$, and

$$
\theta=\frac{\omega}{1-q}+a,
$$

with $\theta \in[a, b]$. The Hahn difference operator was generalized recently in [23] to ${ }_{a} D_{q, \omega}$ defined by

$$
{ }_{a} D_{q, \omega} f(t)= \begin{cases}\frac{f(t)-f(q t+a(1-q)+\omega)}{(t-a)(1-q)-\omega}, & t \neq \theta, \\ f^{\prime}(\theta), & t=\theta,\end{cases}
$$

provided that $f$ is differentiable at $\theta$.

Next, we introduce a new quantum Hahn shifting operator by

$$
{ }_{\theta} \Phi_{q}(m)=q m+(1-q) \theta .
$$

As a special case, if $\omega=a=0$, then (11) is reduced to classical quantum shifting in [1]. If $\omega=0$, then (11) is reduced to the $q$-shifting in (7) studied in [20], and if $a=0$, then (11) is reduced to Hahn shifting as appeared in [10]. In addition, the iterated $k$-times of quantum shifting is defined by

$$
{ }_{\theta} \Phi_{q}^{k}(m)={ }_{\theta} \Phi_{q}^{k-1}\left({ }_{\theta} \Phi_{q}(m)\right)=q^{k} m+\left(1-q^{k}\right) \theta
$$

with ${ }_{\theta} \Phi_{q}^{0}(m)=m$.

Proposition 1 The following relations hold:

(i) $(1-q)(t-a)-\omega=(1-q)(t-\theta)=t-{ }_{\theta} \Phi_{q}(t)$;

(ii) ${ }_{\theta} \Phi_{q}(\theta)=\theta$;

(iii) $\left(1-q^{k}\right) a+\omega[k]_{q}=\left(1-q^{k}\right) \theta$, where $\left[k_{q}\right]=\left(1-q^{k}\right) /(1-q), k=0,1,2, \ldots$

The next definition modifies the definition (10) (studied in [23]), taking into account Proposition 1(i)-(iii). 
Definition 1 Let $f$ be a function defined on $[a, b]$. The quantum Hahn difference operator is defined by

$$
{ }_{a} D_{q, \omega} f(t)= \begin{cases}\frac{f(t)-f\left({ }_{\theta} \Phi_{q}(t)\right)}{t-{ }_{-} \Phi_{q}(t)}, & t \neq \theta, \\ f^{\prime}(\theta), & t=\theta,\end{cases}
$$

provided that $f$ is differentiable at $\theta$.

Definition 2 Assume $f:[a, b] \rightarrow \mathbb{R}$ is a given function and consider two points $c, d \in$ $[a, b]$. The $q, \omega$-quantum Hahn integral of $f$ from $c$ to $d$ is defined by

$$
\int_{c}^{d} f(s)_{a} d_{q, \omega} s:=\int_{\theta}^{d} f(s)_{a} d_{q, \omega} s-\int_{\theta}^{c} f(s)_{a} d_{q, \omega} s,
$$

where

$$
\int_{\theta}^{t} f(s)_{a} d_{q, \omega} s=\left[t-{ }_{\theta} \Phi_{q}(t)\right] \sum_{i=0}^{\infty} q^{i} f\left({ }_{\theta} \Phi_{q}^{i}(t)\right)
$$

for $t \in[a, b]$, provided that the series converges at $t=c$ and $t=d$.

Let us define the $\theta$-power function as

$$
(n-m)_{\theta}^{(0)}=1, \quad(n-m)_{\theta}^{(k)}=\prod_{i=0}^{k-1}\left(n-{ }_{\theta} \Phi_{q}^{i}(m)\right), \quad k \in \mathbb{N} \cup\{\infty\} .
$$

For example, $(n-m))_{\theta}^{(4)}=(n-m)\left(n-{ }_{\theta} \Phi_{q}(m)\right)\left(n-{ }_{\theta} \Phi_{q}^{2}(m)\right)\left(n-{ }_{\theta} \Phi_{q}^{3}(m)\right)$. More generally, if $\gamma \in \mathbb{R}$, then

$$
(n-m)_{\theta}^{(\gamma)}=\prod_{i=0}^{\infty} \frac{\left(n-{ }_{\theta} \Phi_{q}^{i}(m)\right)}{\left(n-{ }_{\theta} \Phi_{q}^{\gamma+i}(m)\right)}
$$

with ${ }_{\theta} \Phi_{q}^{\gamma}(m)=q^{\gamma} m+\left(1-q^{\gamma}\right) \theta, \gamma \in \mathbb{R}$. For example,

$$
(n-m))_{\theta}^{\left(\frac{3}{2}\right)}=\frac{(n-m)\left(n-{ }_{\theta} \Phi_{q}(m)\right)\left(n-{ }_{\theta} \Phi_{q}^{2}(m)\right) \cdots}{\left(n-{ }_{\theta} \Phi_{q}^{\frac{3}{2}}(m)\right)\left(n-{ }_{\theta} \Phi_{q}^{\frac{5}{2}}(m)\right)\left(n-{ }_{\theta} \Phi_{q}^{\frac{7}{2}}(m)\right) \cdots} .
$$

Let us state the definitions of Riemann-Liouville type of fractional derivative and integral of quantum Hahn calculus and also Caputo type fractional derivative, which can be found in [24].

Definition 3 The fractional quantum Hahn difference of Riemann-Liouville type of order $v \geq 0$ on an interval $[a, b]$ is defined by $\left({ }_{a} D_{q, \omega}^{0} f\right)(t)=f(t)$ and

$$
\left({ }_{a} D_{q, \omega}^{v} f\right)(t)=\frac{1}{\Gamma_{q}(l-v)}{ }_{a} D_{q, \omega}^{l} \int_{a}^{t}\left(t-{ }_{\theta} \Phi_{q}(s)\right)_{\theta}^{(l-v-1)} f(s)_{a} d_{q, \omega} s, \quad v>0,
$$

where $l$ is the smallest integer greater than or equal to $v$. 
Definition 4 Let $v \geq 0$ and $f$ be a function defined on $[a, b]$. The fractional quantum Hahn integral of Riemann-Liouville type is given by $\left({ }_{a} I_{q, \omega}^{0} f\right)(t)=f(t)$ and

$$
\left({ }_{a} I_{q, \omega}^{v} f\right)(t)=\frac{1}{\Gamma_{q}(v)} \int_{a}^{t}\left(t-{ }_{\theta} \Phi_{q}(s)\right)_{\theta}^{(v-1)} f(s)_{a} d_{q, \omega} s, \quad v>0, t \in[a, b] .
$$

Definition 5 The fractional quantum Hahn difference of Caputo type $v \geq 0$ on an interval $[a, b]$ is defined by $\left({ }_{a}^{c} D_{q, \omega}^{0} f\right)(t)=f(t)$ and

$$
\left({ }_{a}^{c} D_{q, \omega}^{v} f\right)(t)=\frac{1}{\Gamma_{q}(l-v)} \int_{a}^{t}\left(t-{ }_{\theta} \Phi_{q}(s)\right)_{\theta}^{(l-v-1)}{ }_{a} D_{q, \omega}^{l} f(s)_{a} d_{q, \omega} s, \quad v>0
$$

where $l$ is the smallest integer greater than or equal to $v$.

If $\omega=0$, then $\theta=a$ and the above fractional quantum Hahn calculus is reduced to fractional quantum calculus on the interval $[a, b]$ as appeared in [22].

Theorem 1 ([24]) Let $\alpha, \beta \in \mathbb{R}^{+}, \lambda \in(-1, \infty)$ and $\theta \in[a, b]$. The following formulas hold:

(i) $\left({ }_{a} I_{q, \omega}^{\alpha}(x-a)_{\theta}^{(\lambda)}\right)(t)=\frac{\Gamma_{q}(\lambda+1)}{\Gamma_{q}(\alpha+\lambda+1)}(t-a)_{\theta}^{(\alpha+\lambda)}$;

(ii) $\left({ }_{a} D_{q, \omega}^{\alpha}(x-a)_{\theta}^{(\lambda)}\right)(t)=\frac{\Gamma_{q}(\lambda+1)}{\Gamma_{q}(\lambda-\alpha+1)}(t-a)_{\theta}^{(\lambda-\alpha)}$.

Theorem 2 ([24]) Let $f(t)$ be a function defined on an interval $[a, b], \beta, v \in \mathbb{R}^{+}, \alpha \in(N-$ $1, N)$ and $\theta \in[a, b]$. Then, we have:

(i) $\left({ }_{a} I_{q, \omega}^{\beta} I_{q, \omega}^{v} f\right)(t)=\left({ }_{a} I_{q, \omega}^{v} I_{q, \omega}^{\beta} f\right)(t)=\left({ }_{a} I_{q, \omega}^{\beta+v} f\right)(t)$;

(ii) $\left({ }_{a} D_{q, \omega a}^{\beta} I_{q, \omega}^{\beta} f\right)(t)=\left({ }_{a}^{c} D_{q, \omega a}^{\beta} I_{q, \omega}^{\beta} f\right)=f(t)$;

(iii) $\left({ }_{a} I_{q, \omega}^{\alpha} a D_{q, \omega}^{\alpha} f\right)(t)=f(t)+c_{1}(t-a)_{\theta}^{(\alpha-1)}+c_{2}(t-a)_{\theta}^{(\alpha-2)}+\cdots+c_{N}(t-a)_{\theta}^{(\alpha-N)}$;

(iv) $\left({ }_{a} I_{q, \omega a}^{\alpha}{ }^{c} D_{q, \omega}^{\alpha} f\right)(t)=f(t)+d_{0}+d_{1}(t-a)_{\theta}^{(1)}+d_{2}(t-a)_{\theta}^{(2)}+\cdots+d_{N-1}(t-a)_{\theta}^{(N-1)}$, for some $c_{i}, d_{j} \in \mathbb{R}, i=1,2, \ldots, N, j=0,1, \ldots, N-1$.

The rest of the paper is organized as follows: In Sect. 2.1 we prove the existence and uniqueness results for the the impulsive Hahn difference boundary value problem (1), while the corresponding results for the impulsive Hahn difference boundary value problem (2) are presented in Sect. 2.2. Examples illustrating the obtained results are presented in Sect. 3.

\section{Impulsive fractional Hahn difference equations}

To establish our results, we define intervals $J_{k}=\left[t_{k}, t_{k+1}\right), k=0,1,2, \ldots, m-1, J_{m}=\left[t_{m}, T\right]$ and $J=[0, T]$, with impulsive points $0=t_{0}<t_{1}<\cdots<t_{k}<t_{k+1}<\cdots<t_{m}<t_{m+1}=T$. In addition, we define the space $P C(J, \mathbb{R})=\{x: J \rightarrow \mathbb{R}: x(t)$ is continuous everywhere except for some $t_{k}$ at which $x\left(t_{k}^{+}\right)$and $x\left(t_{k}^{-}\right)$exist and $\left.x\left(t_{k}^{+}\right)=x\left(t_{k}\right), k=1,2, \ldots, m\right\}$. Observe that $P C(J, \mathbb{R})$ is a Banach space equipped with the norm $\|x\|=\sup \{|x(t)|: t \in J\}$. From Sect. 1 , we replace all parameters, $a, q, \omega$ and $v$ of fractional quantum Hahn calculus in Definitions $3-5$ by $t_{k}, q_{k}, \omega_{k}$ and $v_{k}, k=0,1,2, \ldots, m$, respectively. Also we assume that

$$
\theta_{k}=\frac{\omega_{k}}{1-q_{k}}+t_{k} \in J_{k}, \quad k=0,1,2, \ldots, m .
$$


In the next subsections, the fractional quantum Hahn calculus is used to establish the existence and uniqueness results for the impulsive fractional Hahn difference boundary value problems (1) and (2).

\subsection{Impulsive problem of fractional quantum Hahn difference equation of order \\ $0<v_{k} \leq 1$}

In this subsection, we investigate the impulsive Hahn difference boundary value problem (1).

The following lemma deals with the linear variant of problem (1) and gives a representation of the solution.

Lemma 1 Let $\xi_{1}+\xi_{2} \neq 0$ and $h \in C(J, \mathbb{R})$ be a given function. Then, the function $x$ is a solution of the impulsive Hahn difference boundary value problem

$$
\left\{\begin{array}{l}
c_{t_{k}}^{c} D_{q_{k}, \omega_{k}}^{v_{k}} x(t)=h(t), \quad t \in J_{k}, k=0,1,2, \ldots, m, \\
\Delta x\left(t_{k}\right)=\varphi_{k}\left(x\left(t_{k}^{-}\right)\right), \quad k=1,2, \ldots, m, \\
\xi_{1} x(0)+\xi_{2} x(T)=\xi_{3}
\end{array}\right.
$$

if and only if

$$
\begin{aligned}
x(t)= & \frac{\xi_{3}}{\xi_{1}+\xi_{2}}-\frac{\xi_{2}}{\xi_{1}+\xi_{2}}\left[\sum_{i=0}^{m}\left(t_{i} I_{q_{i}, \omega_{i}}^{v_{i}} h\right)\left(t_{i+1}^{-}\right)+\sum_{j=1}^{m} \varphi_{j}\left(x\left(t_{j}^{-}\right)\right)\right] \\
& +\sum_{i=0}^{k-1}\left(t_{i} I_{q_{i}, \omega_{i}}^{v_{i}} h\right)\left(t_{i+1}^{-}\right)+\sum_{j=1}^{k} \varphi_{j}\left(x\left(t_{j}^{-}\right)\right)+\left({ }_{t_{k}} I_{q_{k}, \omega_{k}}^{v_{k}} h\right)(t),
\end{aligned}
$$

for $t \in J_{k}, k=0,1,2, \ldots, m$, with $\sum_{a}^{b}(\cdot)=0$, when $b<a$.

Proof Applying Theorem 2(iv), for $t \in J_{0}$, we obtain

$$
{ }_{t} I_{q_{0}, \omega_{0}}^{\nu_{0}}\left({ }_{t_{0}}^{c} D_{q_{0}, \omega_{0}}^{\nu_{0}} x\right)(t)=x(t)=c_{0}+\left(t_{0} I_{q_{0}, \omega_{0}}^{\nu_{0}} h\right)(t)
$$

for some $c_{0} \in \mathbb{R}$. In particular, when $t=t_{1}^{-}$, it follows that

$$
x\left(t_{1}^{-}\right)=c_{0}+\left(t_{0} I_{q_{0}, \omega_{0}}^{\nu_{0}} h\right)\left(t_{1}^{-}\right) .
$$

For $t \in J_{1}$, using the same process, we have

$$
x(t)=x\left(t_{1}\right)+\left({ }_{t_{1}} I_{q_{1}, \omega_{1}}^{\nu_{1}} h\right)(t) .
$$

The impulsive condition, $x\left(t_{1}\right)=x\left(t_{1}^{-}\right)+\varphi_{1}\left(x\left(t_{1}^{-}\right)\right)$, yields

$$
\begin{aligned}
x(t) & =x\left(t_{1}^{-}\right)+\varphi_{1}\left(x\left(t_{1}^{-}\right)\right)+\left(t_{1} I_{q_{1}, \omega_{1}}^{\nu_{1}} h\right)(t) \\
& =c_{0}+\left(t_{t_{0}} \nu_{q_{0}, \omega_{0}}^{\nu_{0}} h\right)\left(t_{1}^{-}\right)+\varphi_{1}\left(x\left(t_{1}^{-}\right)\right)+\left({ }_{t_{1}} I_{q_{1}, \omega_{1}}^{\nu_{1}} h\right)(t) .
\end{aligned}
$$


Repeating the above argument, for $t \in J_{k}, k=0,1,2, \ldots, m$, we get

$$
x(t)=c_{0}+\sum_{i=0}^{k-1}\left({ }_{t} I_{q_{i}, \omega_{i}}^{v_{i}} h\right)\left(t_{i+1}^{-}\right)+\sum_{j=1}^{k} \varphi_{j}\left(x\left(t_{j}^{-}\right)\right)+\left({ }_{t_{k}} I_{q_{k}, \omega_{k}}^{v_{k}} h\right)(t)
$$

with $\sum_{a}^{b}(\cdot)=0$, when $b<a$. Since $x(0)=c_{0}$ and

$$
x(T)=c_{0}+\sum_{i=0}^{m}\left({ }_{t_{i}} I_{q_{i}, \omega_{i}}^{v_{i}} h\right)\left(t_{i+1}^{-}\right)+\sum_{j=1}^{m} \varphi_{j}\left(x\left(t_{j}^{-}\right)\right),
$$

with $t_{m+1}^{-}=T$, we can compute, with boundary condition in (16), that

$$
c_{0}=\frac{\xi_{3}}{\xi_{1}+\xi_{2}}-\frac{\xi_{2}}{\xi_{1}+\xi_{2}}\left[\sum_{i=0}^{m}\left({ }_{t_{i}} I_{q_{i}, \omega_{i}}^{\nu_{i}} h\right)\left(t_{i+1}^{-}\right)+\sum_{j=1}^{m} \varphi_{j}\left(x\left(t_{j}^{-}\right)\right)\right] .
$$

Substituting the constant $c_{0}$ in the integral equation (18), we obtain the desired result in (17). The converse follows by direct computation. The proof is completed.

In the following, for convenience we use the abbreviation

$$
\begin{aligned}
\left({ }_{t_{k}} I_{q_{k}, \omega_{k}}^{v_{k}} f(s, x(s))\right)(t) & =\frac{1}{\Gamma_{q_{k}}\left(v_{k}\right)} \int_{t_{k}}^{t}\left(t-\theta_{\theta_{k}} \Phi_{q_{k}}(s)\right)_{\theta_{k}}^{\left(v_{k}-1\right)} f(s, x(s))_{t_{k}} d_{q_{k}, \omega_{k}} s \\
& =\left({ }_{t_{k}} I_{q_{k}, \omega_{k}}^{v_{k}} f_{x}\right)(t),
\end{aligned}
$$

for $k=0,1,2, \ldots, m$, and put

$$
\begin{aligned}
& \Lambda_{1}=\left(1+\frac{\left|\xi_{2}\right|}{\left|\xi_{1}+\xi_{2}\right|}\right)\left(L_{1} \sum_{i=0}^{m} \frac{\left(t_{i+1}-t_{i}\right)_{\theta_{i}}^{\left(v_{i}\right)}}{\Gamma_{q_{i}}\left(v_{i}+1\right)}+\frac{L_{2}}{2} m(m+1)\right) \\
& \Lambda_{2}=\frac{\left|\xi_{3}\right|}{\left|\xi_{1}+\xi_{2}\right|}+\left(1+\frac{\left|\xi_{2}\right|}{\left|\xi_{1}+\xi_{2}\right|}\right)\left(M \sum_{i=0}^{m} \frac{\left(t_{i+1}-t_{i}\right)_{\theta_{i}}^{\left(v_{i}\right)}}{\Gamma_{q_{i}}\left(v_{i}+1\right)}+\frac{N}{2} m(m+1)\right) .
\end{aligned}
$$

Now, we are in the position to establish the existence of a unique solution of problem (1) by using the Banach contraction mapping principle.

Theorem 3 Letf $: J \times \mathbb{R} \rightarrow \mathbb{R}$ and $\varphi_{k}: \mathbb{R} \rightarrow \mathbb{R}, k=1,2, \ldots, m$, be given functions satisfying

$$
|f(t, x)-f(t, y)| \leq L_{1}|x-y|, \quad L_{1}>0, \forall t \in J, x, y \in \mathbb{R},
$$

and

$$
\left|\varphi_{k}(x)-\varphi_{k}(y)\right| \leq L_{2}|x-y|, \quad L_{2}>0, \forall x, y \in \mathbb{R}
$$

If

$$
\Lambda_{1}<1
$$

then problem (1) has a unique solution on $J$. 
Proof In view of Lemma 1, we transform the boundary value problem (1), into an operator equation $x(t)=A x(t)$, where $A: P C(J, \mathbb{R}) \rightarrow P C(J, \mathbb{R})$ is defined by

$$
\begin{aligned}
A x(t):= & \frac{\xi_{3}}{\xi_{1}+\xi_{2}}-\frac{\xi_{2}}{\xi_{1}+\xi_{2}}\left[\sum_{i=0}^{m}\left(t_{i} I_{q_{i}, \omega_{i}}^{\nu_{i}} f_{x}\right)\left(t_{i+1}^{-}\right)+\sum_{j=1}^{m} \varphi_{j}\left(x\left(t_{j}^{-}\right)\right)\right] \\
& +\sum_{i=0}^{k-1}\left({ }_{t_{i}}{ }_{q_{i}, \omega_{i}}^{\nu_{i}} f_{x}\right)\left(t_{i+1}^{-}\right)+\sum_{j=1}^{k} \varphi_{j}\left(x\left(t_{j}^{-}\right)\right)+\left({ }_{t_{k}} I_{q_{k}, \omega_{k}}^{v_{k}} f_{x}\right)(t), \quad t \in J .
\end{aligned}
$$

Also we define a set $B_{r}$ by $B_{r}=\{x \in P C(J, \mathbb{R}):\|x\| \leq r\}$ where $r>\Lambda_{2} /\left(1-\Lambda_{1}\right)$. It should be shown that $A B_{r} \subset B_{r}$. Setting $\sup _{t \in J}\left|f_{0}\right|=M$, $\max _{j}\left|\varphi_{j}(0)\right|=N$, where $f_{0}=f(t, 0)$, and using $\left|f_{x}\right| \leq\left|f_{x}-f_{0}\right|+\left|f_{0}\right|$ and $\left|\varphi_{j}(x)\right| \leq\left|\varphi_{j}(x)-\varphi_{j}(0)\right|+\left|\varphi_{j}(0)\right|, j=1,2,3, \ldots, m$, for any $x \in B_{r}$, we have

$$
\begin{aligned}
|A x(t)| & \\
\leq & \frac{\left|\xi_{3}\right|}{\left|\xi_{1}+\xi_{2}\right|}+\frac{\left|\xi_{2}\right|}{\left|\xi_{1}+\xi_{2}\right|}\left[\sum_{i=0}^{m}\left({ }_{t_{i}} I_{q_{i}, \omega_{i}}^{v_{i}}\left|f_{x}\right|\right)\left(t_{i+1}^{-}\right)+\sum_{j=1}^{m}\left|\varphi_{j}\left(x\left(t_{j}^{-}\right)\right)\right|\right] \\
& +\sum_{i=0}^{k-1}\left(t_{t_{i}} I_{q_{i}, \omega_{i}}^{v_{i}}\left|f_{x}\right|\right)\left(t_{i+1}^{-}\right)+\sum_{j=1}^{k}\left|\varphi_{j}\left(x\left(t_{j}^{-}\right)\right)\right|+\left(t_{k} I_{q_{k}, \omega_{k}}^{v_{k}}\left|f_{x}\right|\right)(t) \\
\leq & \frac{\left|\xi_{3}\right|}{\left|\xi_{1}+\xi_{2}\right|}+\frac{\left|\xi_{2}\right|}{\left|\xi_{1}+\xi_{2}\right|}\left[\sum_{i=0}^{m}\left({ }_{t_{i}} I_{q_{i}, \omega_{i}}^{v_{i}}\left(\left|f_{x}-f_{0}\right|+\left|f_{0}\right|\right)\right)\left(t_{i+1}^{-}\right)\right. \\
& \left.+\sum_{j=1}^{m}\left(\left|\varphi_{j}\left(x\left(t_{j}\right)\right)-\varphi_{j}(0)\right|+\left|\varphi_{j}(0)\right|\right)\right]+\sum_{i=0}^{k-1}\left(t_{i} I_{q_{i}, \omega_{i}}^{\nu_{i}}\left(\left|f_{x}-f_{0}\right|+\left|f_{0}\right|\right)\right)\left(t_{i+1}^{-}\right) \\
& +\sum_{j=1}^{k}\left(\left|\varphi_{j}\left(x\left(t_{j}\right)\right)-\varphi_{j}(0)\right|+\left|\varphi_{j}(0)\right|\right)+\left(t_{k} I_{q_{k}, \omega_{k}}^{v_{k}}\left(\left|f_{x}-f_{0}\right|+\left|f_{0}\right|\right)\right)(t) \\
\leq & \frac{\left|\xi_{3}\right|}{\left|\xi_{1}+\xi_{2}\right|}+\frac{\left|\xi_{2}\right|}{\left|\xi_{1}+\xi_{2}\right|}\left[\left(L_{1} r+M\right) \sum_{i=0}^{m}\left(t_{i} I_{q_{i}, \omega_{i}}^{v_{i}}(1)\right)\left(t_{i+1}^{-}\right)+\left(L_{2} r+N\right) \sum_{j=1}^{m}(1)\right] \\
& +\left(L_{1} r+M\right) \sum_{i=0}^{m}\left(t_{i} I_{q_{i}, \omega_{i}}^{v_{i}}(1)\right)\left(t_{i+1}^{-}\right)+\left(L_{2} r+N\right) \sum_{j=1}^{m}(1) \\
= & \Lambda_{1} r+\Lambda_{2}<r .
\end{aligned}
$$

This show that $\|A x\| \leq r$, which leads to $A B_{r} \subset B_{r}$. Now, we will prove that the operator $A$ is a contraction by using (21). For any $x, y \in B_{r}$, we have

$$
\begin{aligned}
& |A x(t)-A y(t)| \\
& \leq \frac{\left|\xi_{2}\right|}{\left|\xi_{1}+\xi_{2}\right|}\left[\sum_{i=0}^{m}\left(\left(_{t_{i}} I_{q_{i}, \omega_{i}}^{v_{i}}\left|f_{x}-f_{y}\right|\right)\left(t_{i+1}^{-}\right)+\sum_{j=1}^{m}\left|\varphi_{j}\left(x\left(t_{j}^{-}\right)\right)-\varphi_{j}\left(y\left(t_{j}^{-}\right)\right)\right|\right]\right. \\
& \quad+\sum_{i=0}^{k-1}\left(t_{i} I_{q_{i}, \omega_{i}}^{\nu_{i}}\left|f_{x}-f_{y}\right|\right)\left(t_{i+1}^{-}\right)+\sum_{j=1}^{k}\left|\varphi_{j}\left(x\left(t_{j}^{-}\right)\right)-\varphi_{j}\left(y\left(t_{j}^{-}\right)\right)\right|
\end{aligned}
$$




$$
\begin{aligned}
& +\left(t_{k} I_{q_{k}, \omega_{k}}^{v_{k}}\left|f_{x}-f_{y}\right|\right)(t) \\
\leq & L_{1}\|x-y\|\left(\frac{\left|\xi_{2}\right|}{\left|\xi_{1}+\xi_{2}\right|} \sum_{i=0}^{m}\left(t_{i} I_{q_{i}, \omega_{i}}^{v_{i}}(1)\right)\left(t_{i+1}^{-}\right)+\sum_{i=0}^{m}\left(t_{i} I_{q_{i}, \omega_{i}}^{v_{i}}(1)\right)\left(t_{i+1}^{-}\right)\right) \\
& +L_{2}\|x-y\|\left(\frac{\left|\xi_{2}\right|}{\left|\xi_{1}+\xi_{2}\right|}+1\right) \sum_{j=1}^{m}(1) \\
= & \Lambda_{1}\|x-y\|,
\end{aligned}
$$

which yields $\|A x-A y\| \leq \Lambda_{1}\|x-y\|$. From (21), we conclude that the operator $A$ is a contraction on $B_{r}$. By the Banach contraction mapping principle, therefore, the impulsive boundary value problem of fractional quantum Hahn difference equation (1) has a unique solution $x$ on $J$ such that $\|x\| \leq r$. The proof is complete.

Corollary 1 Let constants $\xi_{1} \neq 0$ and $\xi_{2}=0$ in (1), then we have the impulsive initial value problem

$$
\left\{\begin{array}{l}
t_{k} D_{q_{k}, \omega_{k}}^{v_{k}} x(t)=f(t, x(t)), \quad t \in J_{k}, k=0,1,2, \ldots, m \\
\Delta x\left(t_{k}\right)=\varphi_{k}\left(x\left(t_{k}^{-}\right)\right), \quad k=1,2, \ldots, m \\
x(0)=\frac{\xi_{3}}{\xi_{1}}
\end{array}\right.
$$

If the functions $f$ and $\varphi_{i}, i=1,2, \ldots, m$, satisfy (19) and (20), respectively, and if

$$
L_{1} \sum_{i=0}^{m} \frac{\left(t_{i+1}-t_{i}\right)_{\theta_{i}}^{\left(v_{i}\right)}}{\Gamma_{q_{i}}\left(v_{i}+1\right)}+\frac{L_{2}}{2} m(m+1)<1,
$$

then the impulsive initial value problem of fractional quantum Hahn difference equation (22) has a unique solution on J.

\subsection{Impulsive problem of fractional quantum Hahn difference equation of order $1<v_{k} \leq 2$}

Consider now the impulsive fractional Hahn difference boundary value problem (2).

Lemma 2 Let $g \in C(J, \mathbb{R})$. Then, the function $x$ is a solution of the impulsive Hahn difference boundary value problem

$$
\left\{\begin{array}{l}
t_{k} D_{q_{k}, \omega_{k}}^{v_{k}} x(t)=g(t), \quad t \in J_{k}, k=0,1,2, \ldots, m, \\
\Delta x\left(t_{k}\right)=\varphi_{k}\left(x\left(t_{k}^{-}\right)\right), \quad k=1,2, \ldots, m, \\
t_{k} D_{q_{k}, \omega_{k}} x\left(t_{k}^{+}\right)-t_{k-1} D_{q_{k-1}, \omega_{k-1}} x\left(t_{k}^{-}\right)=\varphi_{k}^{*}\left(x\left(t_{k}^{-}\right)\right), \quad k=1,2, \ldots, m, \\
x(0)=\eta_{1}, \quad t_{m} D_{q_{m}, \omega_{m}} x(T)=\eta_{2},
\end{array}\right.
$$


if and only if

$$
\begin{aligned}
x(t)= & \eta_{1}+\left(\eta_{2}-\sum_{j=0}^{m}\left(t_{j} I_{q_{j}, \omega_{j}}^{\nu_{j}-1} g\right)\left(t_{j+1}^{-}\right)-\sum_{i=1}^{m} \varphi_{i}^{*}\left(x\left(t_{i}^{-}\right)\right)\right) \sum_{i=0}^{k}\left(\left[t_{i+1}\right]-t_{i}\right) \\
& +\sum_{i=0}^{k-1}\left[\left(t_{i} I_{q_{i}, \omega_{i}}^{\nu_{i}} g\right)\left(t_{i+1}^{-}\right)+\varphi_{i+1}\left(x\left(t_{i+1}^{-}\right)\right)\right] \\
& +\sum_{i=1}^{k}\left\{\left(\left[t_{i+1}\right]-t_{i}\right) \sum_{j=0}^{i-1}\left[\left(t_{j} I_{q_{j}, \omega_{j}}^{\nu_{j}-1} g\right)\left(t_{j+1}^{-}\right)+\varphi_{j+1}^{*}\left(x\left(t_{j+1}^{-}\right)\right)\right]\right\} \\
& +\left(t_{k} I_{q_{k}, \omega_{k}}^{\nu_{k}} g\right)(t),
\end{aligned}
$$

for $t \in J_{k}, k=0,1,2, \ldots, m$, with $\sum_{a}^{b}(\cdot)=0$, when $b<a$ and

$$
\left[t_{i+1}\right]= \begin{cases}t_{i+1}, & t_{i+1} \leq t_{k}, \\ t, & t_{i+1}>t_{k} .\end{cases}
$$

Proof Taking the Riemann-Liouville fractional quantum Hahn integral of order $v_{0}$ to the first equation in (23) and applying Theorem 2(iv), for $t \in J_{0}$, we get

$$
{ }_{t} I_{q_{0}, \omega_{0}}^{v_{0}}\left({ }_{t_{0}}^{c} D_{q_{0}, \omega_{0}}^{v_{0}} x\right)(t)=x(t)=c_{0}+c_{1}\left(t-t_{0}\right)_{\theta_{0}}^{(1)}+\left(t_{t_{0}} I_{q_{0}, \omega_{0}}^{v_{0}} g\right)(t) .
$$

From the first condition, $x(0)=\eta_{1}$, we have $c_{0}=\eta_{1}$ and from (15) with $k=1$, for $t=t_{1}^{-}$, we obtain

$$
x\left(t_{1}^{-}\right)=\eta_{1}+c_{1}\left(t_{1}-t_{0}\right)+\left({ }_{t_{0}} I_{q_{0}, \omega_{0}}^{\nu_{0}} g\right)\left(t_{1}^{-}\right),
$$

with $(a-b)_{\theta}^{(1)}=(a-b), a, b \in \mathbb{R}$. In addition, we can formulate from (25) that

$$
{ }_{t} D_{q_{0}, \omega_{0}} x(t)=c_{1}+\left({ }_{t_{0}} I_{q_{0}, \omega_{0}}^{\nu_{0}-1} g\right)(t)
$$

and then $t_{0} D_{q_{0}, \omega_{0}} x\left(t_{1}^{-}\right)=c_{1}+\left({ }_{t_{0}} I_{q_{0}, \omega_{0}}^{\nu_{0}-1} g\right)\left(t_{1}^{-}\right)$.

For $t \in\left[t_{1}, t_{2}\right)=J_{1}$, we have

$$
x(t)=x\left(t_{1}^{+}\right)+{ }_{t_{1}} D_{q_{1}, \omega_{1}} x\left(t_{1}^{+}\right)\left(t-t_{1}\right)+\left({ }_{t_{1}} I_{q_{1}, \omega_{1}}^{\nu_{1}} g\right)(t) .
$$

Since

$$
\begin{aligned}
x\left(t_{1}^{+}\right) & =x\left(t_{1}^{-}\right)+\varphi_{1}\left(x\left(t_{1}^{-}\right)\right) \\
& =\eta_{1}+c_{1}\left(t_{1}-t_{0}\right)+\left(t_{0} I_{q_{0}, \omega_{0}}^{\nu_{0}} g\right)\left(t_{1}^{-}\right)+\varphi_{1}\left(x\left(t_{1}^{-}\right)\right),
\end{aligned}
$$

and

$$
\begin{aligned}
{ }_{t_{1}} D_{q_{1}, \omega_{1}} x\left(t_{1}^{+}\right) & ={ }_{t_{0}} D_{q_{0}, \omega_{0}} x\left(t_{1}^{-}\right)+\varphi_{1}^{*}\left(x\left(t_{1}^{-}\right)\right) \\
& =c_{1}+\left({ }_{t_{0}} I_{q_{0}, \omega_{0}}^{\nu_{0}-1} g\right)\left(t_{1}^{-}\right)+\varphi_{1}^{*}\left(x\left(t_{1}^{-}\right)\right),
\end{aligned}
$$


then (27) can be written as

$$
\begin{aligned}
x(t)= & \eta_{1}+c_{1}\left(t_{1}-t_{0}\right)+\left({ }_{t_{0}} I_{q_{0}, \omega_{0}}^{\nu_{0}} g\right)\left(t_{1}^{-}\right)+\varphi_{1}\left(x\left(t_{1}^{-}\right)\right) \\
& +\left(t-t_{1}\right)\left[c_{1}+\left({ }_{t_{0}} I_{q_{0}, \omega_{0}}^{\nu_{0}-1} g\right)\left(t_{1}^{-}\right)+\varphi_{1}^{*}\left(x\left(t_{1}^{-}\right)\right)\right]+\left({ }_{t_{1}} I_{q_{1}, \omega_{1}}^{v_{1}} g\right)(t) .
\end{aligned}
$$

Repeating the above process, for $t \in J_{k}$, we get

$$
\begin{aligned}
x(t)= & \eta_{1}+c_{1} \sum_{i=0}^{k}\left(\left[t_{i+1}\right]-t_{i}\right)+\sum_{i=0}^{k-1}\left[\left(t_{i} I_{q_{i}, \omega_{i}}^{v_{i}} g\right)\left(t_{i+1}^{-}\right)+\varphi_{i+1}\left(x\left(t_{i+1}^{-}\right)\right)\right] \\
& +\sum_{i=1}^{k}\left\{\left(\left[t_{i+1}\right]-t_{i}\right) \sum_{j=0}^{i-1}\left[\left({ }_{t_{j}} I_{q_{j}, \omega_{j}}^{v_{j}-1} g\right)\left(t_{j+1}^{-}\right)+\varphi_{j+1}^{*}\left(x\left(t_{j+1}^{-}\right)\right)\right]\right\} \\
& +\left(t_{k} I_{q_{k}, \omega_{k}}^{v_{k}} g\right)(t) .
\end{aligned}
$$

To compute $c_{1}$, we have

$$
\eta_{2}={ }_{t_{m}} D_{q_{m}, \omega_{m}} x(T)=c_{1}+\sum_{j=0}^{m}\left({ }_{t_{j}} I_{q_{j}, \omega_{j}}^{\nu_{j}-1} g\right)\left(t_{j+1}^{-}\right)+\sum_{i=1}^{m} \varphi_{i}^{*}\left(x\left(t_{i}^{-}\right)\right),
$$

which leads to

$$
c_{1}=\eta_{2}-\sum_{j=0}^{m}\left({ }_{t j} I_{q_{j}, \omega_{j}}^{\nu_{j}-1} g\right)\left(t_{j+1}^{-}\right)-\sum_{i=1}^{m} \varphi_{i}^{*}\left(x\left(t_{i}^{-}\right)\right) .
$$

Therefore, the result in (24) holds when substituting the constant $c_{1}$ in (28). The converse follows by direct computation. This competes the proof.

To accomplish our goal, we define the operator $G: P C(J, \mathbb{R}) \rightarrow P C(J, \mathbb{R})$ by

$$
\begin{aligned}
G x(t)= & \eta_{1}+\left(\eta_{2}-\sum_{j=0}^{m}\left({ }_{j} I_{q_{j}, \omega_{j}}^{v_{j}-1} f_{x}\right)\left(t_{j+1}^{-}\right)-\sum_{i=1}^{m} \varphi_{i}^{*}\left(x\left(t_{i}^{-}\right)\right)\right) \sum_{i=0}^{k}\left(\left[t_{i+1}\right]-t_{i}\right) \\
& +\sum_{i=0}^{k-1}\left[\left(t_{i} I_{q_{i}, \omega_{i}}^{v_{i}} f_{x}\right)\left(t_{i+1}^{-}\right)+\varphi_{i+1}\left(x\left(t_{i+1}^{-}\right)\right)\right] \\
& +\sum_{i=1}^{k}\left\{\left(\left[t_{i+1}\right]-t_{i}\right) \sum_{j=0}^{i-1}\left[\left(t_{j} I_{q_{j}, \omega_{j}}^{\nu_{j}-1} f_{x}\right)\left(t_{j+1}^{-}\right)+\varphi_{j+1}^{*}\left(x\left(t_{j+1}^{-}\right)\right)\right]\right\} \\
& +\left(t_{k} I_{q_{k}, \omega_{k}}^{v_{k}} f_{x}\right)(t) .
\end{aligned}
$$

The Banach fixed point theorem and Leray-Schauder's nonlinear alternative will be used to study the existence and uniqueness results for the impulsive Hahn difference boundary 
value problem (2). Now, we set

$$
\begin{aligned}
& \Lambda_{3}=T \sum_{i=0}^{m} \frac{\left(t_{i+1}-t_{i}\right)_{\theta_{i}}^{\left(v_{i}-1\right)}}{\Gamma_{q_{i}}\left(v_{i}\right)}+\sum_{i=0}^{m} \frac{\left(t_{i+1}-t_{i}\right)_{\theta_{i}}^{\left(v_{i}\right)}}{\Gamma_{q_{i}}\left(v_{i}+1\right)}+\sum_{i=1}^{m}\left(t_{i+1}-t_{i}\right) \sum_{j=0}^{i-1} \frac{\left(t_{j+1}-t_{j}\right)_{\theta_{j}}^{\left(v_{j}-1\right)}}{\Gamma_{q_{j}}\left(v_{j}\right)}, \\
& \Lambda_{4}=m T+\sum_{i=1}^{m}\left(t_{i+1}-t_{i}\right) i, \quad \Lambda_{5}=L_{1} \Lambda_{3}+L_{2} m+L_{3} \Lambda_{4}, \quad \Lambda_{6}=\left|\eta_{1}\right|+\left|\eta_{2}\right| T .
\end{aligned}
$$

Theorem 4 Let $f$ and $\varphi_{k}$ be given functions satisfying (19) and (20), respectively, for all $k=1,2, \ldots, m$. Assume the $\varphi_{k}^{*}: \mathbb{R} \rightarrow \mathbb{R}$ such that

$$
\left|\varphi_{k}^{*}(x)-\varphi_{k}^{*}(y)\right| \leq L_{3}|x-y|, \quad L_{3}>0, \forall x, y \in \mathbb{R}
$$

If $\Lambda_{5}<1$, then the impulsive fractional quantum Hahn difference boundary value problem (2) has a unique solution on $J$.

Proof The existence of a unique solution for the problem (2) will be proved by considering an operator equation $x=G x$, where $G$ is defined by (29). Consider the set $B_{R}=\{x \in$ $P C(J, \mathbb{R}):\|x\| \leq R\}$, where a positive constant $R$ satisfies

$$
R>\frac{\Lambda_{6}+\Lambda_{3} M+m N+\Lambda_{4} K}{1-\Lambda_{5}},
$$

and $K=\max _{j}\left|\varphi_{j}^{*}(0)\right|$ and constants $M, N$ are defined in the proof of Theorem 3. We claim that $G B_{R} \subset B_{R}$. Since

$$
\begin{aligned}
& |G x(t)| \\
& \leq\left|\eta_{1}\right|+\left(\left|\eta_{2}\right|+\sum_{j=0}^{m}\left(t_{j} I_{q_{j} j \omega_{j}}^{\nu_{j}-1}\left|f_{x}\right|\right)\left(t_{j+1}^{-}\right)+\sum_{i=1}^{m}\left|\varphi_{i}^{*}\left(x\left(t_{i}^{-}\right)\right)\right|\right) \sum_{i=0}^{m}\left(t_{i+1}-t_{i}\right) \\
& \quad+\sum_{i=0}^{m-1}\left[\left(t_{i} I_{q_{i}, \omega_{i}}^{v_{i}}\left|f_{x}\right|\right)\left(t_{i+1}^{-}\right)+\left|\varphi_{i+1}\left(x\left(t_{i+1}^{-}\right)\right)\right|\right] \\
& \quad+\sum_{i=1}^{m}\left\{\left(t_{i+1}-t_{i}\right) \sum_{j=0}^{i-1}\left[\left(t_{j} I_{q_{j}, \omega_{j}}^{\nu_{j}-1}\left|f_{x}\right|\right)\left(t_{j+1}^{-}\right)+\left|\varphi_{j+1}^{*}\left(x\left(t_{j+1}^{-}\right)\right)\right|\right]\right\}+\left(t_{m} I_{q_{m}, \omega_{m}}^{v_{m}}\left|f_{x}\right|\right)(T),
\end{aligned}
$$

and $\left|f_{x}\right| \leq\left|f_{x}-f_{0}\right|+\left|f_{0}\right| \leq L_{1} R+M,\left|\varphi_{j}(x)\right| \leq\left|\varphi_{j}(x)-\varphi_{j}(0)\right|+\left|\varphi_{j}(0)\right| \leq L_{2} R+N,\left|\varphi_{j}^{*}(x)\right| \leq$ $\left|\varphi_{j}^{*}(x)-\varphi_{j}^{*}(0)\right|+\left|\varphi_{j}^{*}(0)\right| \leq L_{3} R+K, j=1,2,3, \ldots, m$, for any $x \in B_{R}$, we have

$$
|G x(t)| \leq \Lambda_{6}+R \Lambda_{5} \Lambda_{3} M+m N+\Lambda_{4} K<R
$$

which yields $\|G x\| \leq R$. To prove the contraction property of operator $G$, for any $x, y \in B_{R}$, we consider the inequalities

$$
\begin{aligned}
& |G x(t)-G y(t)| \\
& \quad \leq T \sum_{j=0}^{m}\left(t_{j} I_{q_{j}, \omega_{j}}^{\nu_{j}-1}\left|f_{x}-f_{y}\right|\right)\left(t_{j+1}^{-}\right)+T \sum_{i=1}^{m}\left|\varphi_{i}^{*}\left(x\left(t_{i}^{-}\right)\right)-\varphi_{i}^{*}\left(y\left(t_{i}^{-}\right)\right)\right|
\end{aligned}
$$




$$
\begin{aligned}
& +\sum_{i=0}^{m-1}\left[\left(t_{i} I_{q_{i}, \omega_{i}}^{v_{i}}\left|f_{x}-f_{y}\right|\right)\left(t_{i+1}^{-}\right)+\left|\varphi_{i+1}\left(x\left(t_{i+1}^{-}\right)\right)-\varphi_{i+1}\left(y\left(t_{i+1}^{-}\right)\right)\right|\right] \\
& +\sum_{i=1}^{m}\left\{( t _ { i + 1 } - t _ { i } ) \sum _ { j = 0 } ^ { i - 1 } \left[\left(t_{j} I_{q_{j}, \omega_{j}}^{\nu_{j}-1}\left|f_{x}-f_{y}\right|\right)\left(t_{j+1}^{-}\right)\right.\right. \\
& \left.\left.+\left|\varphi_{j+1}^{*}\left(x\left(t_{j+1}^{-}\right)\right)-\varphi_{j+1}^{*}\left(y\left(t_{j+1}^{-}\right)\right)\right|\right]\right\}+\left(t_{m} I_{q_{m}, \omega_{m}}^{v_{m}}\left|f_{x}-f_{y}\right|\right)(T) \\
& \leq L_{1}\|x-y\| T \sum_{j=0}^{m}\left(t_{j} I_{q_{j}, \omega_{j}}^{v_{j}-1}(1)\right)\left(t_{j+1}^{-}\right)+L_{3}\|x-y\| T \sum_{i=1}^{m}(1) \\
& +L_{1}\|x-y\| \sum_{i=0}^{m-1}\left(t_{t_{i}} I_{q_{i}, \omega_{i}}^{v_{i}}(1)\right)\left(t_{i+1}^{-}\right)+L_{2}\|x-y\| \sum_{i=0}^{m-1}(1) \\
& +L_{1}\|x-y\| \sum_{i=1}^{m}\left(t_{i+1}-t_{i}\right) \sum_{j=0}^{i-1}\left(t_{j} I_{q_{j}, \omega_{j}}^{\nu_{j}-1}(1)\right)\left(t_{j+1}^{-}\right) \\
& +L_{3}\|x-y\| \sum_{i=1}^{m}\left(t_{i+1}-t_{i}\right) \sum_{j=0}^{i-1}(1)+L_{1}\|x-y\|\left(t_{m} I_{q_{m}, \omega_{m}}^{v_{m}}(1)\right)(T) \\
& =L_{1} \Lambda_{3}\|x-y\|+L_{2} m\|x-y\|+L_{3} \Lambda_{4}\|x-y\| \\
& =\Lambda_{5}\|x-y\| .
\end{aligned}
$$

Then we get $\|G x-G y\| \leq \Lambda_{5}\|x-y\|$ which implies that $G$ is a contraction operator as $\Lambda_{5}<1$. Therefore problem (2) has a unique solution $x$ on $J$.

Lemma 3 (Nonlinear alternative for single valued maps, [25]) Let E be a Banach space, $C$ a closed, convex subset of $E, U$ an open subset of $C$ and $0 \in U$. Suppose that $F: \bar{U} \rightarrow C$ is a continuous, compact (that is, $F(\bar{U})$ is a relatively compact subset of $C$ ) map. Then either

(i) F has a fixed point in $\bar{U}$, or

(ii) There is a $u \in \partial U$ (the boundary of $U$ in $C$ ) and $\lambda \in(0,1)$ with $u=\lambda F(u$ ).

Theorem 5 Assume that the functions $f: J \times \mathbb{R} \rightarrow \mathbb{R}, \varphi_{k}, \varphi_{k}^{*}: \mathbb{R} \rightarrow \mathbb{R}, k=1,2, \ldots, m$, are continuous. In addition, we suppose that:

$\left(H_{1}\right)$ There exist a continuous nondecreasing function $\psi_{1}:[0, \infty) \rightarrow(0, \infty)$ and a continuous function $p: J \rightarrow \mathbb{R}^{+}$such that

$$
|f(t, x)| \leq p(t) \psi_{1}(|x|) \quad \text { for each }(t, x) \in J \times \mathbb{R}
$$

$\left(H_{2}\right)$ There exist continuous nondecreasing functions $\psi_{2}, \psi_{3}:[0, \infty) \rightarrow(0, \infty)$ such that

$$
\left|\varphi_{k}(x)\right| \leq \psi_{2}(|x|) \text { and }\left|\varphi_{k}^{*}(x)\right| \leq \psi_{3}(|x|)
$$

for all $x \in \mathbb{R}, k=1,2, \ldots, m$. 
$\left(H_{3}\right)$ There exists a constant $Q>0$ such that

$$
\frac{Q}{\Lambda_{6}+p^{*} \psi_{1}(Q) \Lambda_{3}+\psi_{2}(Q) m+\psi_{3}(Q) \Lambda_{4}}>1
$$

where $p^{*}=\sup \{p(t): t \in J\}$.

Then the impulsive fractional quantum Hahn difference boundary value problem (2) has at least one solution on $J$.

Proof Let us prove the theorem by applying Lemma 3. For a positive number $\rho$, we define the set $B_{\rho}=\{x \in P C(J, \mathbb{R}):\|x\| \leq \rho\}$. Clearly, $B_{\rho}$ is a closed, convex subset of $P C(J, \mathbb{R})$. Let $\left\{x_{n}\right\}$ be a sequence converging to $x$. Then for $t \in J$, we obtain

$$
\begin{aligned}
& \left|G x_{n}(t)-G x(t)\right| \\
& \leq\left(\sum_{j=0}^{m}\left(t_{j} I_{q_{j}, \omega_{j}}^{v_{j}-1}\left|f_{x_{n}}-f_{x}\right|\right)\left(t_{j+1}^{-}\right)-\sum_{i=1}^{m}\left|\varphi_{i}^{*}\left(x_{n}\left(t_{i}^{-}\right)\right)-\varphi_{i}^{*}\left(x\left(t_{i}^{-}\right)\right)\right|\right) \\
& \quad \times \sum_{i=0}^{k}\left(\left[t_{i+1}\right]-t_{i}\right)+\sum_{i=0}^{k-1}\left[\left(t_{i}{ }_{q_{i}, \omega_{i}}^{\nu_{i}}\left|f_{x_{n}}-f_{x}\right|\right)\left(t_{i+1}^{-}\right)\right. \\
& \left.\quad+\left|\varphi_{i+1}\left(x_{n}\left(t_{i+1}^{-}\right)\right)-\varphi_{i+1}\left(x\left(t_{i+1}^{-}\right)\right)\right|\right]+\sum_{i=1}^{k}\left\{\left(\left[t_{i+1}\right]-t_{i}\right)\right. \\
& \left.\quad \times \sum_{j=0}^{i-1}\left[\left(t_{j} I_{q_{j}, \omega_{j}}^{v_{j}-1}\left|f_{x_{n}}-f_{x}\right|\right)\left(t_{j+1}^{-}\right)+\left|\varphi_{j+1}^{*}\left(x_{n}\left(t_{j+1}^{-}\right)\right)-\varphi_{j+1}^{*}\left(x\left(t_{j+1}^{-}\right)\right)\right|\right]\right\} \\
& \quad+\left(t_{k} I_{q_{k}, \omega_{k}}^{v_{k}}\left|f_{x_{n}}-f_{x}\right|\right)(t) \rightarrow 0, \quad \text { as } n \rightarrow \infty .
\end{aligned}
$$

Hence the operator $G$ is continuous which is one of assumptions in Lemma 3. In the next step, we will prove the compactness of operator $G$.

For $t \in J$ and $x \in B_{\rho}$, we have

$$
\begin{aligned}
& |G x(t)| \\
& \leq\left|\eta_{1}\right|+\left(\left|\eta_{2}\right|+\sum_{j=0}^{m}\left(t_{j} I_{q_{j}, \omega_{j}}^{\nu_{j}-1}\left|f_{x}\right|\right)\left(t_{j+1}^{-}\right)+\sum_{i=1}^{m}\left|\varphi_{i}^{*}\left(x\left(t_{i}^{-}\right)\right)\right|\right) \sum_{i=0}^{k}\left(\left[t_{i+1}\right]-t_{i}\right) \\
& \quad+\sum_{i=0}^{k-1}\left[\left(t_{i} I_{q_{i}, \omega_{i}}^{v_{i}}\left|f_{x}\right|\right)\left(t_{i+1}^{-}\right)+\left|\varphi_{i+1}\left(x\left(t_{i+1}^{-}\right)\right)\right|\right] \\
& \quad+\sum_{i=1}^{k}\left\{\left(\left[t_{i_{i+1}}\right]-t_{i}\right) \sum_{j=0}^{i-1}\left[\left(t_{j} I_{q_{j}, \omega_{j}}^{v_{j}-1}\left|f_{x}\right|\right)\left(t_{j+1}^{-}\right)+\left|\varphi_{j+1}^{*}\left(x\left(t_{j+1}^{-}\right)\right)\right|\right]\right\} \\
& \quad+\left(t_{k} I_{q_{k}, \omega_{k}}^{v_{k}}\left|f_{x}\right|\right)(t) \\
& \leq\left|\eta_{1}\right|+\left(\left|\eta_{2}\right|+p^{*} \psi_{1}(\rho) \sum_{j=0}^{m}\left(t_{j} I_{q_{j}, \omega_{j}}^{\nu_{j}-1}(1)\right)\left(t_{j+1}^{-}\right)+\psi_{3}(\rho) \sum_{i=1}^{m}(1)\right) \sum_{i=0}^{m}\left(t_{i+1}-t_{i}\right) \\
& \quad+p^{*} \psi_{1}(\rho) \sum_{i=0}^{m-1}\left(t_{i} I_{q_{i}, \omega_{i}}^{v_{i}}(1)\right)\left(t_{i_{+1}}^{-}\right)+\psi_{2}(\rho) \sum_{i=1}^{m}(1)
\end{aligned}
$$




$$
\begin{aligned}
& \quad+\sum_{i=1}^{m}\left\{\left(t_{i+1}-t_{i}\right) \sum_{j=0}^{i-1}\left[p^{*} \psi_{1}(\rho)\left({ }_{t_{j}} I_{q_{j}, \omega_{j}}^{v_{j}-1}(1)\right)\left(t_{j+1}^{-}\right)+\psi_{3}(\rho)\right]\right\} \\
& +p^{*} \psi_{1}(\rho)\left(t_{m} I_{q_{m}, \omega_{m}}^{v_{m}}(1)\right)(T) \\
& \leq\left|\eta_{1}\right|+\left|\eta_{2}\right| T+p^{*} \psi_{1}(\rho)\left(T \sum_{j=0}^{m} \frac{\left(t_{j+1}-t_{j}\right)_{\theta_{j}}^{\left(v_{j}-1\right)}}{\Gamma_{q_{j}}\left(v_{j}\right)}+\sum_{i=0}^{m} \frac{\left(t_{i+1}-t_{i}\right)_{\theta_{i}}^{\left(v_{i}\right)}}{\Gamma_{q_{i}}\left(v_{i}+1\right)}\right. \\
& \left.\quad+\sum_{i=1}^{m}\left(t_{i+1}-t_{i}\right) \sum_{j=0}^{i-1} \frac{\left(t_{j+1}-t_{j}\right)_{\theta_{j}}^{\left(v_{j}-1\right)}}{\Gamma_{q_{j}}\left(v_{j}\right)}\right)+\psi_{2}(\rho) m \\
& \quad+\psi_{3}(\rho)\left(m T+\sum_{i=1}^{m}\left(t_{i+1}-t_{i}\right) i\right) \\
& :=K .
\end{aligned}
$$

Hence, we obtain $\|G x\| \leq K$, which means that the set $G B_{\rho}$ is a uniformly bounded set. Next we let $\tau_{1}, \tau_{2} \in J_{k}$ for some $k \in\{0,1,2, \ldots, m\}$ with $\tau_{1}<\tau_{2}$, and let $x \in B_{\rho}$. Then we see that

$$
\begin{aligned}
\left|G x\left(\tau_{2}\right)-G x\left(\tau_{1}\right)\right| \leq & \left(\left|\eta_{2}\right|+p^{*} \psi_{1}(\rho) \sum_{j=0}^{m}\left(t_{i j} I_{q_{j}, \omega_{j}}^{v_{j}-1}(1)\right)\left(t_{j+1}^{-}\right)+\psi_{3}(\rho) m\right)\left|\tau_{2}-\tau_{1}\right| \\
& +\left|\tau_{2}-\tau_{1}\right| \sum_{j=0}^{k-1}\left[p^{*} \psi_{1}(\rho)\left(t_{j} I_{q_{j}, \omega_{j}}^{v_{j}-1}(1)\right)\left(t_{j+1}^{-}\right)+\psi_{3}(\rho)\right] \\
& +p^{*} \psi_{1}(\rho)\left[\left(t_{k} I_{q_{k}, \omega_{k}}^{v_{k}}(1)\right)\left(\tau_{2}\right)-\left(t_{k} I_{q_{k}, \omega_{k}}^{v_{k}}(1)\right)\left(\tau_{1}\right)\right] \\
= & \left(\left|\eta_{2}\right|+p^{*} \psi_{1}(\rho) \sum_{j=0}^{m} \frac{\left(t_{j+1}-t_{j}\right)_{\theta_{j}}^{\left(v_{j}-1\right)}}{\Gamma_{q_{j}}\left(v_{j}\right)}+\psi_{3}(\rho) m\right)\left|\tau_{2}-\tau_{1}\right| \\
& +\left|\tau_{2}-\tau_{1}\right| \sum_{j=0}^{k-1}\left[p^{*} \psi_{1}(\rho) \frac{\left(t_{j+1}-t_{j}\right)_{\theta_{j}}^{\left(v_{j}-1\right)}}{\Gamma_{q_{j}}\left(v_{j}\right)}+\psi_{3}(\rho)\right] \\
& +p^{*} \psi_{1}(\rho)\left[\left(\frac{\left(\tau_{2}-t_{k}\right)_{\theta_{k}}^{\left(v_{k}\right)}}{\Gamma_{q_{k}}\left(v_{k}+1\right)}\right)-\left(\frac{\left(\tau_{1}-t_{k}\right)_{\theta_{k}}^{\left(v_{k}\right)}}{\Gamma_{q_{k}}\left(v_{k}+1\right)}\right)\right] .
\end{aligned}
$$

The right-hand side of the above inequality tends to zero as $\tau_{1} \rightarrow \tau_{2}$ (independently of $x$ ). This shows that the set $G B_{\rho}$ is an equicontinuous set. Therefore the set $G B_{\rho}$ is relatively compact. From the above and Arzelá-Ascoli theorem, the operator $G$ is completely continuous or compact. Hence one more of assumptions of Lemma 3 holds.

The result will follow from Lemma 3 if we can prove the boundedness of the set of all solutions to equations $x=\lambda G x$ for $\lambda \in(0,1)$. Let $x$ be a solution of problem (2). Then, for $t \in J$, we recall the computations in proving that $G$ is bounded. For $\lambda \in(0,1)$, let $x=\lambda G x$. Then we have

$$
|x(t)| \leq \Lambda_{6}+p^{*} \psi_{1}(\|x\|) \Lambda_{3}+\psi_{2}(\|x\|) m+\psi_{3}(\|x\|) \Lambda_{4}
$$


which yields

$$
\frac{\|x\|}{\Lambda_{6}+p^{*} \psi_{1}(\|x\|) \Lambda_{3}+\psi_{2}(\|x\|) m+\psi_{3}(\|x\|) \Lambda_{4}} \leq 1
$$

By assumption $\left(H_{3}\right)$, there exists a positive constant $Q$ such that $\|x\| \neq Q$. Let us define $U=\left\{x \in B_{\rho}:\|x\|<Q\right\}$. It is obvious that $G: \bar{U} \rightarrow P C(J, \mathbb{R})$ is continuous and completely continuous. Therefore, there is no $x \in \partial U$ such that $x=\lambda G x$ for some $\lambda \in(0,1)$. By Lemma 3, thus, we get the result that $G$ has a fixed point $x \in \bar{U}$ which is a solution of problem (2) on $J$. The proof is completed.

\section{Examples}

In this section we give examples to illustrate the usefulness of our main results.

Example 1 Consider the following impulsive fractional quantum Hahn difference boundary value problem:

$$
\left\{\begin{array}{l}
c_{k} D_{\frac{k+1}{k+2}, \frac{k+2}{k+3}}^{\frac{k}{k+5 k+6}} x(t)=\frac{1}{2}\left(\frac{e^{-t}}{t+17}\right)\left(\frac{x^{2}(t)+2|x(t)|}{1+|x(t)|}\right)+\frac{2}{7}, \quad t \in J_{k}, J=[0,4], \\
\Delta x\left(t_{k}\right)=\frac{1}{k+18}\left|\sin x\left(t_{k}^{-}\right)\right|, \quad t_{k}=k, k=1,2,3 \\
\frac{1}{2} x(0)+\frac{2}{3} x(4)=\frac{4}{5} .
\end{array}\right.
$$

Here $v_{k}=(k+2) /(k+3)<1, q_{k}=(k+1) /(k+2), \omega_{k}=1 /\left(k^{2}+5 k+6\right), k=0,1,2,3, m=3$, $T=4, \xi_{1}=1 / 2, \xi_{2}=2 / 3, \xi_{3}=4 / 5$.

In addition, we observe that $\theta_{k}=(1 /(k+3))+k \in J_{k}, k=0,1,2,3$. By using a mathematical program, we can find that

$$
\begin{aligned}
& \left(1+\frac{\left|\xi_{2}\right|}{\left|\xi_{1}+\xi_{2}\right|}\right) \sum_{i=0}^{m} \frac{\left(t_{i+1}-t_{i}\right)_{\theta_{i}}^{\left(v_{i}\right)}}{\Gamma_{q_{i}}\left(v_{i}+1\right)} \approx 8.357863592111553 \\
& \frac{1}{2}\left(1+\frac{\left|\xi_{2}\right|}{\left|\xi_{1}+\xi_{2}\right|}\right) m(m+1) \approx 9.428571428571431
\end{aligned}
$$

Setting

$$
f(t, x)=\frac{1}{2}\left(\frac{e^{-t}}{t+17}\right)\left(\frac{x^{2}+2|x|}{1+|x|}\right)+\frac{2}{7} \quad \text { and } \quad \varphi_{k}(x)=\frac{1}{k+18}|\sin x|
$$

we compute that

$$
|f(t, x)-f(t, y)| \leq(1 / 17)|x-y| \quad \text { and } \quad\left|\varphi_{k}(x)-\varphi_{k}(y)\right| \leq(1 / 19)|x-y| .
$$

Then we get $\Lambda_{1} \approx 0.987879636333851<1$, by using $L_{1}=1 / 17$ and $L_{2}=1 / 19$. Hence, by Theorem 3 , the impulsive fractional quantum Hahn difference boundary value problem (31) has a unique solution on $[0,4]$. 
Example 2 Consider the impulsive fractional quantum Hahn difference boundary value problem

$$
\left\{\begin{array}{l}
c_{t_{k}}^{c} D_{\frac{k+3}{k+5}, \frac{2 k+3}{k+5}}^{\frac{1}{k+2}} x(t)=\frac{1}{(t+10)^{2}}\left(\frac{x^{8}(t)}{\left|x^{7}(t)\right|+1}+1\right), \quad t \in J_{k}, J=[0,4], \\
\Delta x\left(t_{k}\right)=\frac{k^{2}}{10\left(k^{2}+1\right)}\left|x\left(t_{k}^{-}\right)\right|+2, \quad k=1,2,3, t_{k}=k, \\
t_{k} D_{\frac{k+3}{k+5}, \frac{1}{k+5}} x\left(t_{k}^{+}\right)-t_{k-1} D_{\frac{k+2}{k+4}, \frac{1}{k+4}} x\left(t_{k}^{-}\right)=\frac{\sin ^{2} k}{100}\left|x\left(t_{k}^{-}\right)\right|+3, \quad k=1,2,3, t_{k}=k, \\
x(0)=\frac{4}{7}, \quad t_{3} D_{\frac{3}{4}, \frac{1}{8}} x(4)=\frac{5}{9} .
\end{array}\right.
$$

Here $v_{k}=(2 k+3) /(k+2), 1<v_{k} \leq 2, q_{k}=(k+3) /(k+5), \omega_{k}=1 /(k+5), k=0,1,2,3$, $m=3, T=4, \eta_{1}=4 / 7, \eta_{2}=5 / 9$. We find that $\theta_{k}=(1 / 2)+k \in J_{k}, k=0,1,2,3$. By using a mathematical program, we obtain constants as

$$
\Lambda_{3} \approx 40.771217238380451, \quad \Lambda_{4}=18, \quad \Lambda_{6} \approx 2.793650793650793 .
$$

Setting

$$
\begin{aligned}
& f(t, x)=\frac{1}{(t+10)^{2}}\left(\frac{x^{8}}{\left|x^{7}\right|+1}+1\right) \\
& \varphi_{k}(x)=\frac{k^{2}}{10\left(k^{2}+1\right)}|x|+2 \text { and } \varphi_{k}^{*}(x)=\frac{\sin ^{2} k}{100}|x|+3,
\end{aligned}
$$

and choosing $p(t)=\left(1 /(t+10)^{2}\right), \psi_{1}(u)=u+1, \psi_{2}(u)=(1 / 10) u+2$ and $\psi_{3}(u)=(1 / 100) u+3$, it follows that

$$
|f(t, u)| \leq p(t) \psi_{1}(|u|), \quad\left|\varphi_{k}(u)\right| \leq \psi_{2}(|u|), \quad\left|\varphi_{k}^{*}(u)\right| \leq \psi_{3}(|u|),
$$

for all $u \in \mathbb{R}, k=1,2,3$, which imply that conditions $\left(H_{1}\right)-\left(H_{2}\right)$ hold. For $p^{*}=(1 / 100)$, by direct computation, there exists a constant $Q>562.8514177160808$ satisfying the inequality in $\left(H_{3}\right)$. Applying Theorem 5 , we deduce that the impulsive fractional quantum Hahn difference boundary value problem (32) has at least one solution on $[0,4]$.

\section{Funding}

This research was funded by King Mongkut's University of Technology North Bangkok. Contract no. KMUTNB-61-GOV-B-22.

Availability of data and materials

Data sharing not applicable to this article as no data sets were generated or analyzed during the current study.

Competing interests

The authors declare that they have no competing interests.

Authors' contributions

All authors contributed equally to this work. All authors read and approved the final manuscript.

\section{Author details}

${ }^{1}$ Intelligent and Nonlinear Dynamic Innovations Research Center, Department of Mathematics, Faculty of Applied Science, King Mongkut's University of Technology North Bangkok, Bangkok, Thailand. ${ }^{2}$ Department of Mathematics, University of loannina, loannina, Greece. ${ }^{3}$ Nonlinear Analysis and Applied Mathematics (NAAM)-Research Group, Department of Mathematics, Faculty of Science, King Abdulaziz University, Jeddah, Saudi Arabia.

\section{Publisher's Note}

Springer Nature remains neutral with regard to jurisdictional claims in published maps and institutional affiliations. 


\section{References}

1. Jackson, F.H.: q-Difference equations. Am. J. Math. 32, 305-314 (1910)

2. Kac, V., Cheung, P.: Quantum Calculus. Springer, New York (2002)

3. Annaby, M.H., Mansour, Z.S.: q-Fractional Calculus and Equations. Springer, Berlin (2012)

4. Jagerman, D.L.: Difference Equations with Applications to Queues. Dekker, New York (2000)

5. Aldowah, K.A., Malinowska, A.B., Torres, D.F.M.: The power quantum calculus and variational problems. Dyn. Contin. Discrete Impuls. Syst., Ser. B, Appl. Algorithms 19, 93-116 (2012)

6. Birto da Cruz, A.M.C., Martins, N., Torres, D.F.M.: Symmetric differentiation on time scales. Appl. Math. Lett. 26(2), 264-269 (2013)

7. Cruz, B., Artur, M.C.: Symmetric Quantum Calculus. Ph.D. thesis, Aveiro University, Aveiro (2012)

8. Wu, G.C., Baleanu, D.: New applications of the variational iteration method from differential equations to $q$-fractional difference equations. Adv. Differ. Equ. 2013, 21 (2013)

9. Alvarez-Nodarse, R.: On characterization of classical polynomials. J. Comput. Appl. Math. 196, 320-337 (2006)

10. Hahn, W.: Über Orthogonalpolynomen, die q-Differenzengleichungen genügen. Math. Nachr. 2, 4-34 (1949)

11. Brikshavana, T., Sitthiwirattham, T.: On fractional Hahn calculus. Adv. Differ. Equ. 2017, 354 (2017)

12. Costas-Santos, R.S., Marcellan, F:: Second structure relation for q-semiclassical polynomials of the Hahn Tableau. J. Math. Anal. Appl. 329, 206-228 (2007)

13. Foupouagnigni, M.: Laguerre-Hahn orthogonal polynomials with respect to the Hahn operator: fourth-order difference equation for the $r$ th associated and the Laguerre-Freud equations recurrence coefficients. Ph.D. Thesis, Université Nationale du Bénin, Bénin (1998)

14. Kwon, K.H., Lee, D.W., Park, S.B., Yoo, B.H.: Hahn class orthogonal polynomials. Kyungpook Math. J. 38, 259-281 (1998)

15. Sitthiwirattham, T.: Nonlocal three-point boundary value problems for nonlinear second-order Hahn difference equations with two different $q$, $\omega$-derivatives. Adv. Differ. Equ. 2016, 116 (2016)

16. Sriphanomwan, U., Tariboon, J., Patanarapeeler, N., Ntouyas, S.K., Sitthiwirattham, T.: Nonlocal boundary value problems for second order nonlinear Hahn integro-difference equations with Hahn integral boundary conditions. Adv. Differ. Equ. 2017, 170 (2017)

17. Patanarapeelert, N., Brikshavana, T., Sitthiwirattham, T.: On nonlocal Dirichlet boundary value problems for sequential Caputo fractional Hahn integrodifference equations. Bound. Value Probl. 2018, 6 (2018)

18. Patanarapeelert, N., Sitthiwirattham, T.: On nonlocal Robin boundary value problems for Riemann-Liouville fractional Hahn integrodifference equation. Bound. Value Probl. 2018, 46 (2018)

19. Patanarapeelert, N., Sitthiwirattham, T.: Existence results for fractional Hahn difference and fractional Hahn integral boundary value problems. Discrete Dyn. Nat. Soc. 2017, Article ID 7895186 (2017)

20. Tariboon, J., Ntouyas, S.K.: Quantum calculus on finite intervals and applications to impulsive difference equations. Adv. Differ. Equ. 2013, 282 (2013)

21. Ahmad, B., Ntouyas, S.K., Tariboon, J.: Quantum Calculus: New Concepts, Impulsive IVPs and BVPs, Inequalities. Trends in Abstract and Applied Analysis, vol. 4. World Scientific, Singapore (2016)

22. Tariboon, J., Ntouyas, S.K., Agarwal, P.: New concepts of fractional quantum calculus and applications to impulsive fractional q-difference equations. Adv. Differ. Equ. 2015, 18 (2015)

23. Tariboon, J., Ntouyas, S.K., Sudsutad, W.: New concepts of Hahn calculus and impulsive Hahn difference equations. Adv. Differ. Equ. 2016, 255 (2016)

24. Wang, Y., Liu, Y., Hou, C.: New concepts of fractional Hahn's q, $\omega$-derivative of Riemann-Liouville type and Caputo type and applications. Adv. Differ. Equ. 2018, 292 (2018)

25. Granas, A., Dugundji, J.: Fixed Point Theory. Springer, New York (2003)

\section{Submit your manuscript to a SpringerOpen ${ }^{\circ}$ journal and benefit from:}

- Convenient online submission

- Rigorous peer review

- Open access: articles freely available online

- High visibility within the field

Retaining the copyright to your article

Submit your next manuscript at $\gg$ springeropen.com 